\title{
The Value of Using Pulsed Tissue Doppler Mitral Annulus Velocity during Dobutamine Stress Echocardiography for Assessment of Myocardial Viability in Patients with Coronary Artery Disease before Revasularization
}

\begin{abstract}
Background: Assessment of viability could be of significance in ischemic and heart failure patients before deciding for revascularization. The use of Dobutamine stress echocardiography has the disadvantage of subjective visual evaluation of regional wall motion, so, new technologies have been modified; one of these is to assess mitral annular velocity using tissue Doppler imaging.

Aim: The aim of this study was to evaluate the value of Pulsed wave tissue Doppler mitral annulus velocity with dobutamine echocardiography in assessment of myocardial viability and prediction of functional recovery of wall motion abnormalities after revasularization in patients with coronary artery disease.
\end{abstract}

Patients and methods: The study group included 40 patients, selected consecutively from patients presented to Ain Shams university Hospitals with coronary artery disease as proved by diagnostic coronary angiography and recommended for potential coronary revascularization. Each patient underwent baseline transthoracic echocardiography, in addition to low dose dobutamine echocardiography using TDI at mitral annulus in 6 different walls. All patients were subjected to revascularization ( 30 by $\mathrm{PCI}$ and 10 by CABG) then followed up after 6 months by transthoracic echocardiography to assess improvement in EF and SWMA.

Results: Using the 16 segment method, a total of 640 segments were studied: 250 Segments were considered nondysfunctional (39\%), 390 Segments were dysfunctional: of which, severely hypokinetc (216, $55.4 \%$ of abnormal), 158 segments akinetic ( $40.5 \%$ of abnormal), and 16 Segments were dyskinetic (4.1\% of abnormal). The mean SWM index at rest was 1.9 (0.39).Using low dose dobutamine echocardiography, 220 segments were detected to be viable $(56.4 \%)$, while 170 were non-viable $(43.6 \%)$. In order to relate the results of TDI, The 16 segments were reevaluated into 6 walls per patient. Using this method, 240 walls were studied. 19 walls were excluded due to technical difficulties in assessment of TDI. Pulsed wave TDI demonstrated that dysfunctional areas had lower systolic velocities compared to areas considered as normal. Similarly, the increase from baseline to DSE ( $\Delta$ TDI) was higher in nondysfunctional areas vs. dysfunctional areas. However, there was no significant difference in the mean TDI at rest for viable and non-viable walls as detected by dobutamine stress echocardiography. But the increase in TDI velocity with peak dobutamine was significantly more in viable $(1.97 \pm 0.44)$ vs. non-viable $(1.14 \pm 0.54)$ walls, with $\mathrm{p}<0.0001$. The ejection fraction improved from $39.87 \pm 8.22$ (mean + STD) at baseline to $46.13 \pm 8.58$ (mean + STD) at follow-up. The score index of the segmental wall motion during follow up after revascularization was $1.45 \pm 0.29$ (mean+STD). Among the total dysfunctional segments detected at baseline echocardiography, follow-up echo postoperatively showed improvement by $\geq 1$ score in 239 segments $(61.3 \%)$.In the 220 dobutamine positive segments, 182 segments
Volume 3 Issue I - 2015

\begin{abstract}
Adel Shabana, Walaa Adel, Ghada Samir ElShahed,Amr Adel Atteya, Mohamed Gamal Abdel-Bar, Nagwa Nagi El-Mahalawy

Department of Cardiology,Ain Shams University, Egypt
\end{abstract}

Correspondence: Walaa Adel, Cardiology Department, Ain Shams University, 138 Gesr El-Suez Street, Heliopolis, Cairo I I35I, Egypt, Tel 201005546664 ,

Emaildr_walaa_adel@yahoo.com

Received: March 17, 20I5 | Published: June 30, 2015

improved postrevacsularization $(82.7 \%$ ) while 38 did not show improvement $(17.3 \%)$, whereas in the segments designated as dobutamine non-viable, 122 segments were not improved $(71.8 \%)$ and 48 segments $(28.2 \%)$ improved post revascularization. Taking improvement as the gold standard for viability, sensitivity of dobutamine is: $82.7 \%$, specificity $71.8 \%$, PPV $79.1 \%$, NPV $76.3 \%$. Using TDI method, $115(79.3 \%)$ walls were diagnosed as viable by this method, while 30 walls were nonviable $(20.7 \%) .80$ walls $(55.2 \%$ of total studied walls) were detected to be viable by both dobutamine conventional 2D echocardiography and dobutamine TDI echocardiography (systolic wave), On the other hand, 27 walls (18.6\% of total studied walls) were detected to be nonviable by both methods. However, 38 walls $(26.2 \%$ of total abnormal walls) showed discordance between the two methods. The improvement of 74 walls of 80 combined TDI\& DSE positive walls, making a sensitivity of $90.2 \%$, similarly 24 of 27 concordant TDI \& DSE negative walls did not improve, making a specificity of $92.3 \%$.

Conclusion: The current study confirmed the importance of using TDI in different mitral annular sites, as an objective tool in detecting myocardial viability, and to improve the sensitivity and specificity of DSE.

Keywords: myocardial viability, myocardial hibernation, ischemic cardiomyopathy, revascularization tissue doppler imaging, mitral annular velocity, dobutamine stress echocardiography
Abbreviations: SPECT, single-photon emission computed tomography; $\mathrm{CAD}$, coronary artery disease; $\mathrm{EF}$, ejection fraction; TDI, tissue doppler imaging; LVESD, left ventricular end systolic diameter; WMSI, wall motion score index; LVEDD, left ventricular end diastolic diameter; $\mathrm{CABG}$, coronary artery bypass grafting; WMSI, wall motion score index

\section{Introduction}

Coronary artery disease (CAD) remains a principal cause of morbidity and mortality worldwide. ${ }^{1}$ Many subjects with heart failure and underlying CAD have an important amount of viable but dysfunctional myocardium that may restore the ability to contract 
normally if perfusion improves. ${ }^{2}$ This reawakening of myocardium after restoration of blood flow was referred to as "hibernating". ${ }^{-5}$

There are two main theories to explain the pathophysiology of hibetrnation. The first view of adaptation involves dedifferentiation or embryonic regression, the so called "smart heart" hypothesis, ${ }^{6}$ with a down-regulation in energy utilization and an upregulation of stress proteins. ${ }^{7}$ This counterbalances the effects of ischemia but at the cost of an attenuated level of contractile function. ${ }^{8,9}$ The alternative theory is that this is "forced degeneration", supported by the finding that hibernating myocardium also contains apoptotic cells and cells with autophagosomes, lysosomes, and vacuoles. ${ }^{10}$ Whatever the cause, structural remodeling would be essential to restore contractility, thus chronically impaired but viable myocardium may take weeks or months to recover once flow is restored. ${ }^{11}$ Interventions that bring back blood flow to the hibernating myocardium may return the myocytes to their physiologic function and reprogram the cells to normal expression of key proteins. ${ }^{12}$

The differentiation of viable from nonviable myocardium is therefore highly relevant in patients who are being considered for revascularization ${ }^{13}$. Many patients who demonstrate viability associated with severe LV dysfunction may still be candidates for revascularization rather than for cardiac transplantation. ${ }^{14}$

Several Imaging techniques are used to detect viable myocardium, depending on different characteristics of dysfunctional but viable myocardium. The most widely used and available methods are: ${ }^{15,16}$ Nuclear imaging by Single-photon emission computed tomography (SPECT) (evaluating perfusion, cell membrane integrity, and intact mitochondria with thallium or technetium-labeled agents) and Echocardiography with dobutamine (to assess contractile reserve).

In a recent meta-analysis, all available studies of regional left ventricular function in patients with ischemic left ventricular dysfunction before and after revascularization were pooled. ${ }^{17}$ This analysis confirmed and extended the findings of the previous pooled analysis by the same group..$^{18}$ In general, the nuclear imaging techniques had a higher sensitivity and lower specificity than DSE. Regarding prediction of global function improvement, DSE appeared to have the higher specificity, but the differences between techniques were not statistically significant. ${ }^{17}$

Generally, the final endpoint in viability studies is the longterm prognosis. Several studies and metaanalyses have evaluated the prognostic value of viability in relation to therapy. These data consistently showed better prognosis in patients who had viable myocardium and were revascularized, suggesting that revascularization stabilizes the unstable substrate of dysfunctional but viable myocardium. ${ }^{17,19}$

It should be noted, however, that medical therapy was not standardized in the studies analyzed by Allman K et al., ${ }^{19}$ and the adherence to optimal therapy was not adequately described. In the last decade, the medical treatment of heart failure has continued to improve and significant advances have been made in the techniques for coronary revascularization which have reduced intra-procedural and peri-procedural risks. ${ }^{20}$

Consequently, Camici P et al., ${ }^{21}$ pooled the data from 14 non randomized studies found a trend for a survival benefit in patients with $\mathrm{CAD}$ and $\mathrm{LV}$ dysfunction, with viable myocardium, who underwent revascularization compared with patients with viable myocardium treated medically. In the absence of viable myocardium, no clear-cut difference can be observed between treatments despite the fact that advances in both modalities of coronary revascularization procedures have reduced intra-procedural and peri-procedural risks.
Most of these studies were based on retrospective analysis. On the contrary, reviewing the most recent literature, it was observed that the annual mortality rate in patients treated medically appears to be similar regardless of the presence of viability. ${ }^{22}$

In a substudy from STICH trial ${ }^{22} 601$ patients with CAD and LV dysfunction were enrolled in a randomized trial of medical therapy with or without CABG, using SPECT, DSE, or both to assess myocardial viability. The study concluded that although the presence of viable myocardium was associated with a greater probability of survival in patients with CAD and LV dysfunction, however the assessment of myocardial viability did not recognize patients who can benefit from $\mathrm{CABG}$, as compared with medical therapy alone. This finding may reflect the low rates of death among patients with viable myocardium who received medical therapy alone in STICH study ( $\sim 7 \%$ per year), as compared with previously reported rates..$^{22}$ Recently, Gerber et al studied 144 patients with coronary artery disease and myocardial dysfunction and concluded that detection of functional viable myocardium by DE-CMR is an independent predictor of mortality in patients with ischemic LV dysfunction before revascularization. This conclusion may be useful for pre-operative selection of patients for revascularization.$^{23}$

Echocardiography can allow detection of myocardial viability with a rather reasonable accuracy, using pharmacological stress echocardiography. ${ }^{24}$ In patients with jeopardized but viable myocardium, the LV ejection fraction(EF) will show improvement with low-dose dobutamine in direct proportion to the number of segments with contractile reserve. ${ }^{25}$ Dobutamine-induced segmental and global functional recovery correlates well with other, more complex imaging techniques, including PET and thallium scintigraphy. ${ }^{26,27}$ Furthermore, new developments in stress echocardiography can help as adjuvant to improve viability detection. These include contrast echocardiography, tissue Doppler imaging (TDI) and strain, and three-dimensional echocardiography.

The use of TDI for viability prediction at rest has been limited by its lack of site specificity because the segment of interest can be "tethered" by neighboring segments. Some TDI parameters including peak systolic velocity, isovolumetric contraction, and time-topeak systolic velocity have not been shown to consistently predict functional recovery. ${ }^{28,29}$

Myocardial velocity analysis by TDI at rest and during dobutamine stimulation could allow assessment of myocardial viability. ${ }^{30-32}$ Pulsed Doppler tissue velocity analysis has been performed on apical views with analysis of systolic tissue velocities confined to the basal segments. This approach allows assessment of viability for a whole ventricular wall from apex to base. ${ }^{33}$ Pulsed wave TDI has the ability to quantify myocardial wall motion velocities. Several factors can influence TDI measurements, such as the translational and rotational motion of the heart within the thorax, as well as the angle of incidence of the ultrasound beam relative to the axis of myocardial longitudinal movement. By assessing myocardial velocities from the apical views, the effect of translation and rotation of the heart on the measurement of myocardial velocities is minimized. In addition, the apex acts as a fixed reference point facilitating the assessment of contraction and relaxation in the axial plain without the need for angle correction. A possible limitation in the evaluation of TDI results may be tethering between adjacent regions that can influence its ability to localize differences in myocardial velocity. To avoid this effect, tissue Doppler tracings were obtained with the sample positioned near the mitral annulus to assess the vectorial sum of contraction velocities of the longitudinally oriented myocardial fibres between the base and the apex. $^{30}$ 


\section{Aim}

The aim of this study was to evaluate the value of Pulsed wave tissue Doppler mitral annulus velocity with dobutamine echocardiography in assessment of myocardial viability and prediction of functional recovery of wall motion abnormalities after revasularization in patients with coronary artery disease.

\section{Patients and methods}

Our study group was selected consecutively from patients presented with coronary artery disease as proved by diagnostic coronary angiography and recommended for potential coronary revascularization. Inclusion criteria included the presence of significant $(>50 \%)$ reduction in the luminal diameter of a major coronary artery on the basis of recent coronary angiographic results in addition to regional left ventricular wall motion abnormality on the basis of two-dimensional echocardiography. Patients with decompensated heart failure, significant valvular heart disease, technically difficult echocardiography, or with contraindication to dobutamine administration were excluded from the study.

The purpose of this study was explained to all participants and informed consent was taken, approved by ethical committee.

In addition to history taking, physical examination, and ECG, all patients were subjected to the following:

Baseline two-dimensional transthoracic echocardiography was performed for all patients. Images were acquired with the patient in the left lateral decubitus position using a $2.5 \mathrm{MHz}$ transducer attached to a commercially available VINGMED VIVID VI machine equipped with a harmonic imaging capability. The machine was used to measure the left ventricular end diastolic diameter (LVEDD), left ventricular end systolic diameter (LVESD), Ejection fraction (EF), and resting segmental wall motion. For analytical purposes, the left ventricle was divided into the standard 16-segment model recommended by the American Society of Echocardiography. ${ }^{34} \mathrm{~A}$ wall motion score index (WMSI) serving as an index for the extent of dysfunction was derived by dividing the sum of individual segment scores by the number of interpretable segments. ${ }^{34} \mathrm{~A}$ WMSI of 1.00 indicated normal wall motion.

\section{Dobutamine stress echo test}

An intravenous access was secured for eligible patients, three bipolar ECG leads were connected to the patient's chest for continuous monitoring throughout the study and Dobutamine infusion was used in a stepwise manner (resting, 5 and $10 \mathrm{ug} / \mathrm{kg} / \mathrm{min}$ ) during 3minutes intervals. At each dose heart rate and blood pressure were measured.

Standard echocardiographic views (Parasternal long axis, parasternal short axis, apical four chamber view, apical two chamber view) were recorded with the patient in left lateral decubitus at baseline and at peak infusion. Visual analysis of wall motion and thickening was performed using the previously described scoring system and standard 16-segment model recommended by the American Society of Echocardiography. Regional wall motion score improvement by ${ }^{3}$ 1 grade in at least two contiguous nonoverlap segments at any stage of pharmacologic stress infusion compared with the baseline study indicated viability. ${ }^{35,36}$

\section{End points for interrupting the infusion protocols were}

a. Intolerable symptoms as severe headache, severe nausea and vomiting. b. Severe chest pain and/or dyspnea with evidence of clinical ischemia defined as ${ }^{3} 2.0 \mathrm{~mm}$ of additional ST segment depression or elevation in at least two contiguous leads compared with rest \pm new remote wall motion abnormality or worsening contractility in previously asynergic segments.

c. Limiting asymptomatic side effects including hypertension (systolic blood pressure $>220 \mathrm{~mm} \mathrm{Hg}$ and/or diastolic blood pressure $>120 \mathrm{~mm} \mathrm{Hg}$ ), hypotension (relative or absolute i.e. $>30 \mathrm{mmHg}$ decrease in blood pressure), sustained supraventricular tachyarrhythmia particularly atrial fibrillation, ventricular arrhythmias (frequent, polymorphous premature ventricular beats or ventricular tachycardia) and bradyarrhythmias. In the event of demonstrable ischemia, intravenous beta blockers and/ or nitroglycerin were administered.

\section{Dobutamine stress mitral valve annulus pulsed tissue Doppler imaging}

The echocardiography machine was switched to TDI mode to encode myocardial velocities. The systolic mitral annular motion velocities at 6 mitral annular sites were detected as follows: (anteroseptal and inferolateral walls in the apical long axis view, inferoseptal and anterolateral walls in the apical 4 chamber view, and anterior and inferior walls in the apical 2 chamber view). The peak systolic wave was determined at each of these walls, both at rest and at peak dobutamine infusion. The acoustic power and filter frequencies of the ultrasound scan system were set to the lowest values possible and the sample columns (width of approximately $8 \mathrm{~mm}$ ) was set at the mitral annulus. ${ }^{37}$

\section{Revascularization procedure and followup}

The infarct related epicardial coronary artery stenosis was revascularized by either coronary artery bypass grafting (CABG) or by percutaneous transluminal coronary intervention (PCI). Follow up transthoracic two dimensional echocardiogragraphy and TDI was done after 6 months of the revascularization to detect improvement in regional wall motion and the left ventricular function compared to the basal resting echocardiographic study.

The results were tabulated and statistically analyzed. Specificity and Sensitivity of dobutamine mitral annular tissue Doppler imaging (TDI) AND dobutamine echo were calculated and compared against the gold standard of viability (improvement with revascularization).

\section{Statistical analysis}

Categorical variables will be expressed as number (\%) and continuous variables will be expressed as mean \pm SD. The independent sample t-test and ANOVA will be used to compare the mean values of different groups. Linear regression will be used for correlation analyses, which were expressed as Pearson correlation coefficients. For all tests, $\mathrm{p}$ value $<0.05$ will be considered statistically significant. All the analyses will be performed with commercially available software (SPSS version 19.0; SPSS, Inc., Chicago, IL, USA).

\section{Results}

The study included 40 patients with documented significant coronary artery disease by coronary angiography and scheduled for elective revascularization, by PCI or CABG. Table $1 \& 2$ shows basic characteristics, echocardiography and coronary angiography data. Regional wall motion abnormalities (dysfunctional segments) were present in all patients, with a mean \pm SD of: $9.75 \pm 3.5$ dysfunctional segments per patient. Mean hypokinetic segments/patient $=5.4 \pm 2.9$, akinetic $=3.9 \pm 2.3$, while mean dyskinetic/patient $=0.4 \pm 0.9$. 
Using the 16 segment method, a total of 640 segments were studied: 250 Segments were considered normal or mildly hypokinetc (nondysfunctional) (39\%), 390 Segments were dysfunctional: of which, severely hypokinetc $(216,55.4 \%$ of abnormal and $33.75 \%$ of all), 158 segments akinetic (40.5\% of abnormal, $24.7 \%$ of all), and 16 Segments were dyskinetic (4.1\% of abnormal, and $2.5 \%$ of all). The mean SWM index at rest was $1.9(0.39)$.

Table I Summarizes the basic demographic and echocardiographic characteristics of the study patients

\begin{tabular}{ll}
\hline Parameter & Value \\
\hline Age Mean \pm SD & $49 \pm 8.7$ \\
Males No. (\%) & $34(85 \%)$ \\
Smokers No. (\%) & $29(72.5 \%)$ \\
Diabetic No. (\%) & $23(57.5 \%)$ \\
Hypertension No. (\%) & $32(80 \%)$ \\
Positive family history No. (\%) & $15(37.5 \%)$ \\
Overweight No. (\%) & $27(57.5 \%)$ \\
NSTEMI no. (\%) & $5(12.5 \%)$ \\
STEMI no. (\%) & $22(55 \%)$ \\
CHF & $19(47.5 \%)$ \\
Baseline Echocardiographic Parameters & \\
LVEDD Mean $\pm S D$ & $58.9 \pm 7.9$ \\
LVESD Mean $\pm S D$ & $44.5 \pm 8.9$ \\
IVS Mean $\pm S D$ & $10.6 \pm 2.2$ \\
PW Mean $\pm S D$ & $10.7 \pm 2$ \\
EF Mean $\pm S D$ & $39.9 \pm 8.2$ \\
\hline
\end{tabular}

Table 2 Results of Coronary Angiography

\begin{tabular}{lll}
\hline Angiographic Findings & Number of Patients & Percentage \\
\hline I Vessel Disease & 10 & $25 \%$ \\
2Vessel Disease & 19 & $47.5 \%$ \\
3 Vessel Disease & 11 & $27.5 \%$ \\
Site of lesion & Number of patients & Percentage \\
LM & 4 & $10 \%$ \\
LAD & 27 & $67.5 \%$ \\
LtCx & 29 & $72.5 \%$ \\
RCA & 23 & $57.5 \%$ \\
\hline
\end{tabular}

\section{Detection of myocardial viability}

Detection of viability was done to the 40 patients by using conventional Low dose dobutamine 2D echocardiography and Tissue Doppler at mitral annulus (TDI). The normokinetic segments at rest detected by 2D conventional echocardiography were excluded from the study, so the remaining diseased walls were 390 .

The ejection fraction at rest was $39.87 \pm 8.22$ (mean $+\mathrm{SD}$ ), while during maximal rate of dobutamine infusion during the study was $45.2 \pm 8.4$ (mean $+\mathrm{SD})$. ( $\mathrm{P}$ value $<0.0001)$. The score index of the segmental wall motion at rest was $1.9 \pm 0.3$ (mean $+\mathrm{SD}$ ), while during maximal rate of dobutamine infusion during the study was $1.55 \pm 0.31$ (mean $+\mathrm{SD})$. (P value $<0.0001)$.

Among the 390 affected walls, 220 segments were detected to be viable $(56.4 \%)$, while 170 were non-viable (43.6\%). Among the hypokinetic segments, 120 segments were viable by DSE, representing $55.6 \%$ of hypokinetic segments, while 96 segments were viable among the akinetic segments $(60.8 \%)$ and 4 of the 16 dyskinetic segments were viable, representing $25 \%$ of dyskinetic segments (Figure 1). The mean viability index per patient (number of viable segments divided by number of dysfunctional segments) was 0.56 .

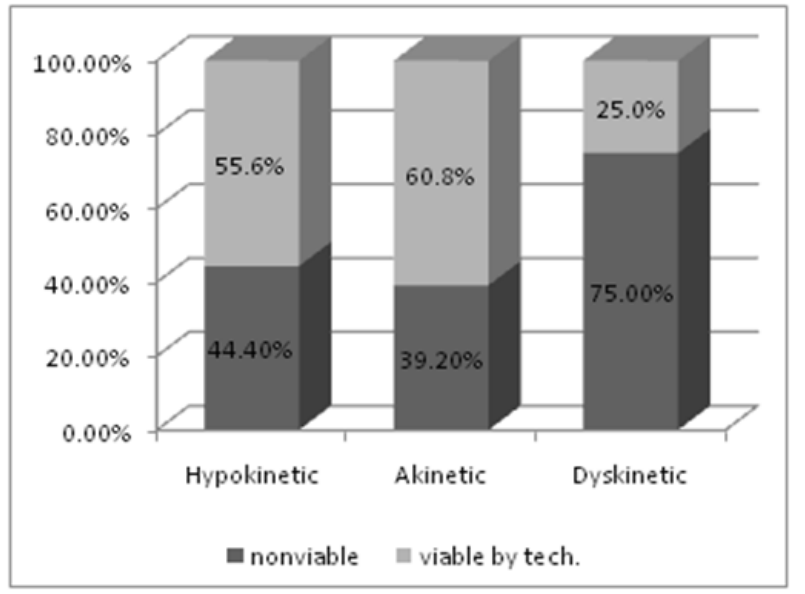

Figure I showing the percentage of viable and nonviable segments in hypokinetic, akinetic and dyskinetic areas, as assessed by DSE.

In order to relate the results of TDI, we reevaluated the 16 segments, where Wall motion was scored by the pattern displayed by two thirds of the sub segments, making 6 walls per patient. ${ }^{32}$ Using this method, 240 walls were studied. Nineteen walls were excluded due to technical difficulties in assessment of TDI, ( 2 in anterior, 2 in anteroseptal, 6 inferoseptal, 2 inferoir, 3 anterolateral, 4 inferolateral) this makes the feasibility of these walls $95 \%, 95 \%, 85 \%, 95 \%$, $92.5 \%$, and $90 \%$, respectively. Analysis of the pulsed-wave Doppler tissue sampling velocity profile showed a significant morphological variation for each wall and with dobutamine.

There were 76 walls (31.7\%) defined as normal/mild hypokinetic walls; 13 in anterior, 12 in anteroseptal, 12 inferoseptal, 14 inferoir, 10 anterolateral and 15 inferolateral. The remaining 145 walls $(60.4 \%)$ were assessed by both DSE and TDI systolic velocity: Using DSE, viability was detected in 86 walls $(59.3 \%)$, while 59 segments were nonviable (40.7\%).

There was variability in mean TDI for each wall (Table 3). Pulsed wave TDI demonstrated that dysfunctional areas had lower systolic velocities compared to areas considered as normal (5.86 \pm 0.7 vs $7.41 \pm 0.76, \mathrm{p}<0.0001)$. This result was similar in each wall separately as in the following table. Similarly, the increase from baseline to low dose DOB. ( $\triangle \mathrm{TDI})$, was higher in nondysfunctional areas vs. dysfunctional areas: This was true in all walls $(2.49 \pm 0.41$ for nondysfunctional vs., $1.55 \pm 0.63$ in dysfunctional areas, $\mathrm{p}<0.0001$ ) and in each wall separately as in the table.

However, There was no significant difference in the mean TDI at rest for viable and non-viable walls as detected by dobutamine stress echocardiography $(5.83 \pm 0.73$ in viable vs. $5.85 \pm 0.76$ in nonviable walls, with $\mathrm{p}=0.9$ nonsignifcant). But the increase in TDI velocity with peak dobutamine ( $\Delta$ TDI) was significantly more in viable $(1.97 \pm 0.44)$ vs. non-viable $(1.14 \pm 0.54)$ walls, with $p<0.0001$. This was statistically significant for the overall walls as well as for each wall site separately (Figure $2 \&$ Table 4).

\section{Assessment post-revascularization}

Thirty patients $(75 \%)$ underwent PCI, while 10 patients $(25 \%)$ underwent CABG. No major acute coronary events were detected in the candidates post revascularization during the followup period. At followup, The LV EF at rest was $46.13 \pm 8.58$ (mean + SD). P value was $<0.0001$, compared to baseline EF). The score index of the segmental wall motion at rest measured during follow up after revascularization 
was $1.45 \pm 0.29$ (mean $+\mathrm{STD}$ ). P value was $<0.0001$, compared to baseline SWMI).

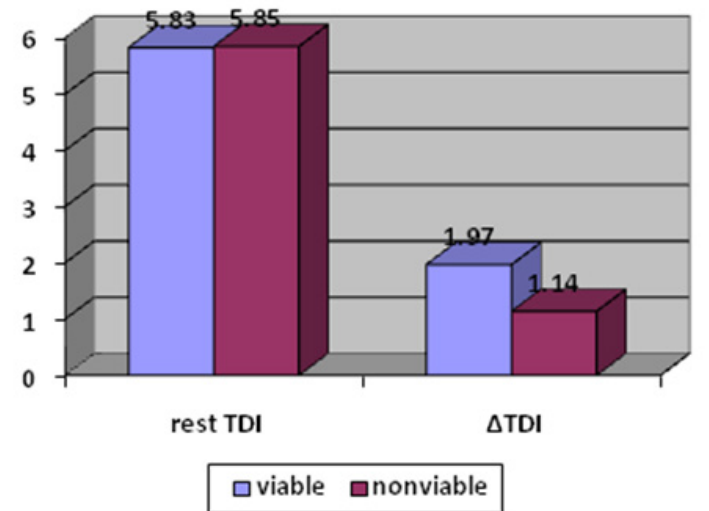

Figure 2 Showing mean TDI and $\triangle T D I$ in viable and nonviable areas by DSE.

Among the total dysfunctional segments detected at baseline echocardiography, follow-up echo postoperatively showed improvement by $\geq 1$ score in 239 segments, representing $61.3 \%$. Mean No. of segments improved per patient 5.9. On analysis, 126 of 216 hypokinetic segments improved post revascularization (58.3\%), while in the 158 akinetic segments, 107 segments improved (67.7\%). Six of the 16 dyskinetic segments improved postrevascularization (37.5\%).

\section{Value of DSE and TDI in Relation to Post- Revascularization Echocardiography}

In the 220 dobutamine positive segments, 182 segments improved postrevacsularization $(82.7 \%$ ) while 38 did not show improvement $(17.3 \%)$, whereas in the segments designated as dobutamine nonviable, 122 segments were not improved (71.8\%) and 48 segments $(28.2 \%)$ improved post revascularization (Figure 3). Taking improvement as the gold standard for viability, sensitivity of dobutamine is: $82.7 \%$, specificity $71.8 \%$, PPV79.1, NPV 76.3 .

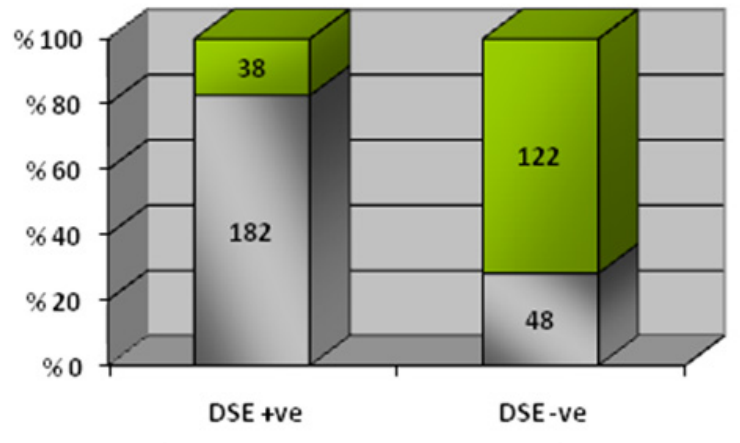

口improved $\square$ not improved

Figure 3 Relation between DSE viability and postoperative improvement in all dysfunctional segments.

On analysis of different types of regional wall motion abnormalities; for hypokinetic segments, sensitivity is 82.5 , specificity: 71.9 , ppv: 78.6, npv: 76.7 For Akinetic segments, sensitivity: $91.7 \%$, specificity 69.4\%, PPV: $82.2 \%$, NPV: $84.3 \%$. Finally, for dyskinetic segments, sensitivity $100 \%$, specificity $83.3 \%$, PPV $66.7 \%$, NPV $100 \%$.

A cutoff value of $\geq 1 \mathrm{~cm} / \mathrm{sec}$ in systolic velocity at low dose DSE was used to define viability of dysfunctional segments. ${ }^{32,38}$ This made $115(79.3 \%)$ walls to be diagnosed as viable by this method, while 30 walls were nonviable $(20.7 \%)$. Eighty walls $(55.2 \%$ of total studied walls) were detected to be viable by both dobutamine conventional 2D echocardiography and dobutamine TDI echocardiography, this represents $93 \%$ of the total number of walls found to be viable by the dobutamine conventional echocardiography and $71.4 \%$ of the walls viable by TDI. On the other hand, 27 walls $(18.6 \%$ of total studied walls) were detected to be non-viable by both dobutamine conventional 2D echocardiography and dobutamine TDI echocardiography, this represents $45.8 \%$ of the total number of walls discovered to be nonviable by the DSE. However, 38 walls ( $26.2 \%$ of total abnormal walls) showed discordance between the two methods (Table 5 \& Figure 4).

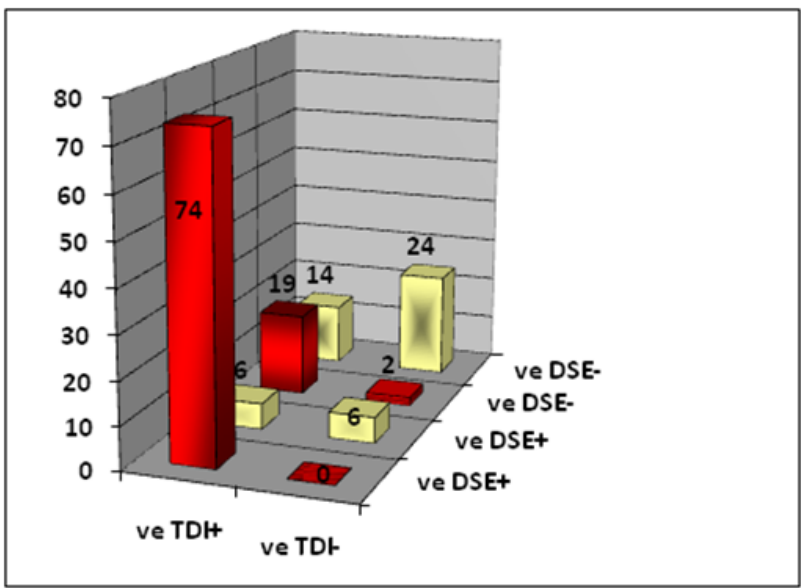

Figure 4 Showing concordance and discordance between DSE [six walls] and TDI systolic wave in relation to improvement of dysfunctional segments post revascularization.

The most significant finding in this table was the improvement of 74 walls of 80 combined TDI\& DSE positive walls, making a sensitivity of $90.2 \%$, similarly 24 of 27 concordant TDI \& DSE negative walls did not improve, making a specificity of $92.3 \%$.

\section{Discussion}

\section{SWM score/EF improvement}

In the present study, the EF at rest was $39.87 \pm 8.22 \%$ ( mean \pm $\mathrm{SD}$ ), while during maximal rate of dobutamine infusion during the study was $45.2 \pm 8.4 \%$ (mean $\pm \mathrm{SD})$. The score index of the segmental wall motion at rest was $1.9 \pm 0.39$ (mean $\pm \mathrm{SD}$ ), while during maximal rate of dobutamine infusion during the study was $1.55 \pm 0.31$ (mean $\pm \mathrm{SD}$ ). At follow up, The LV EF at rest was $46.13 \pm 8.58$ (mean $+\mathrm{SD}$ ). $(\mathrm{P}<0.0001)$. The score index of the segmental wall motion at rest measured during follow up after revascularization was $1.45 \pm 0.29$ (mean + STD). $(\mathrm{P}<0.0001)$.

In other studies, similar results were obtained: Carluccio $E$ et al., ${ }^{36}$ showed that low-dose dobutamine infusion elicited a substantial improvement of both $\mathrm{EF}$ (from $32 \pm 7$ to $41 \pm 8 \%, \mathrm{P}<0.0002$ ) and WMSI (from $2.45 \pm 0.33$ to $1.85 \pm 0.36, \mathrm{P}<0.0002$ ). Similarly, In the study by Carluccio E et al., ${ }^{39}$ where Patients were reevaluated $7.6 \pm$ 3.3 months after revascularization, revascularization significantly improved LV function, ejection fraction increased from $33 \pm 6 \%$ to $45 \pm 10 \%(\mathrm{p}<0.0001)$ and WMSI from $2.29 \pm 0.31$ to $1.74 \pm 0.42$ (p $<0.0001)$.

\section{Low Dose DSE}

In the present study, low dose dobutamine had sensitivity: $82.7 \%$, specificity $71.8 \%$, PPV 79.1 , NPV 76.3 in predicting regional improvement after revascularization. 
The Value of Using Pulsed Tissue Doppler Mitral Annulus Velocity during Dobutamine Stress Echocardiography for Assessment of Myocardial Viability in Patients with Coronary Artery Disease before

Table 3 TDI for each wall, in dysfunctional \&non-dysfunctional areas

\begin{tabular}{lllll}
\hline TDI/Wall & Mean TDI(SD) & $\begin{array}{l}\text { Mean TDI in Normal } \\
\text { Areas (SD) }\end{array}$ & $\begin{array}{l}\text { Mean TDI in } \\
\text { Dysfunctional Areas(SD) Dysfunctional Areas }\end{array}$ \\
\hline Anterior & $6.34(I . I 2)$ & $7.33(I .09)$ & $5.93(0.86)$ & $<0.000 I$ \\
Anteroseptal & $6.39(I .06)$ & $7.58(0.79)$ & $5.86(0.58)$ & $<0.000 I$ \\
Inferoseptal & $6.36(I .05)$ & $7.62(0.77)$ & $5.87(0.67)$ & $<0.000 I$ \\
Inferior & $6.26(I . I)$ & $7.34(0.54)$ & $5.49(0.77)$ & $<0.0001$ \\
Anterolateral & $6.27(0.94)$ & $7.28(0.83)$ & $5.87(0.64)$ & $<0.0001$ \\
inferolateral & $6.42(0.99)$ & $7.31(0.55)$ & $5.95(0.92)$ & $<0.0001$ \\
\hline
\end{tabular}

Table 4 showing TDI change ( $\Delta T D I)$ in normal and abnormal areas in different walls

\begin{tabular}{llll}
\hline TDI/Wall suit & Normal Areas Mean (SD) & $\begin{array}{l}\text { Dysfunctional Areas } \\
\text { Mean (SD) }\end{array}$ & P Value \\
\hline Anterior & $2.32(0.34)$ & $1.89(0.32)$ & 0.003 \\
Anteroseptal & $2.68(0.38)$ & $1.83(0.37)$ & $<0.0001$ \\
Inferoseptal & $2.43(0.34)$ & $1.87(0.67)$ & 0.014 \\
Inferior & $2.53(0.39)$ & $2.1(0.27)$ & 0.002 \\
Anterolateral & $2.52(0.53)$ & $1.8(0.32)$ & 0.003 \\
Inferolateral & $2.47(0.45)$ & $1.74(0.77)$ & 0.015 \\
\hline
\end{tabular}

Table 5 Showing concordance and discordance between DSE [six walls] and TDI systolic wave in relation to improvement of dysfunctional segments post revascularization

\begin{tabular}{|c|c|c|c|c|c|c|c|}
\hline & \multicolumn{3}{|c|}{ Positive DSE } & \multicolumn{3}{|c|}{ Negative DSE } & \multirow{2}{*}{ Tota } \\
\hline & Improved & Not Improved & Total & Improved & Not Improved & Total & \\
\hline +ve TDI & 74 & 6 & 80 & 19 & 14 & 33 & 113 \\
\hline TDI-ve & 0 & 6 & 6 & 2 & 24 & 26 & 32 \\
\hline Total & 74 & 12 & 86 & 21 & 38 & 59 & 145 \\
\hline
\end{tabular}

This is comparable with the meta-analysis by Schinkel A et al., ${ }^{17}$ where Subanalysis of the pooled data showed that low-dose dobutamine echocardiography (33 studies, 1121 patients) had a sensitivity and specificity of 79 and $78 \%$, with a PPV and NPV of 76 and $82 \%$ in prediction of recovery of regional function ${ }^{17}$. However sensitivity is significantly more in our study which may be attributable to different kinds of patients in the different studies involved in the meta-analysis.

In another metanalysis by Camici $\mathrm{P}$ et al. ${ }^{21}$, low dose dobutamine stress had $76 \%$ sensitivity, $81 \%$ specificity, $66 \%$ PPV and $89 \%$ NPV ${ }^{21}$. Similarly, comparing the present study with some of the available individual studies; La Canna G et al., ${ }^{40}$ Studied 28 patients with coronary artery disease and LV dysfunction $(\mathrm{EF} \leq 50)$, low dose dobutamine, was found to have sensitivity of 74, specificity 74 at 12 months post revascularization. ${ }^{40}$ Aggeli C et al. ${ }^{41}$ in a study on Forty-one patients with coronary artery disease and left ventricular dysfunction (ejection fraction $\leq 40 \%$ ), at follow up after revascularization, found low dose dob to have sensitivity $78 \%$, specificity $85 \%$, PPV 87 , NPV $76 \% .{ }^{41}$ Tani $\mathrm{T}$ et al., ${ }^{42}$ investigated the agreement between low-dose dobutamine stress echocardiography and (FDG-PET) and compared each technique's ability to detect myocardial viability and predict functional recovery in 30 patients who underwent revascularization, followed by echocardiography $5 \pm 3$ months : Low dose dobutamine echo was found to have sensitivity $84 \%$, specificity $80 \%$, PPV $88 \%$, NPV $75 \%{ }^{42}$. Karabinos I et al. ${ }^{43}$ evaluated the diagnostic accuracy of DSE in detecting myocardial viability in subgroup of 86 patients with known coronary artery disease before undergoing PTCA with stent implantation. DSE was found to have sensitivity $90 \%$, specificity $100 \%$, positive predictive value $100 \%$, and negative predictive value $91 \%$ in the detection of myocardial viability. ${ }^{43}$

The difference in the results between these studies, including our study, may be related to different patients' characteristics, different inclusion and exclusion criteria, different revascularization procedures and different time for follow up echo post revascularization. Also, in the present study, there was a relatively high incidence of akinetic segments ( $40 \%$ of all dysfunctional segments), which may have more structural changes including fibrosis, cellular dedifferentiation, and loss of contractile elements ${ }^{44}$ than less dysfunctional myocardium. It is expected that such segments may require longer times for functional recovery following revascularization ${ }^{45,46}$ and our single postoperative follow up at six months may have underestimated the amount of recoverable LV dysfunction.

\section{Tissue Doppler}

Several studies in the past have shown that pulsed wave TDI is an effective method to assess quantitatively the existence of myocardial viability after myocardial infarction.

In our study, Pulsed wave TDI demonstrated that dysfunctional walls had significantly lower systolic velocities compared to walls considered as normal. (5.86 \pm 0.7 vs. $7.41 \pm 0.76, p<0.0001)$. Similarly, the increase from baseline to DSE was higher in nondysfunctional walls vs. dysfunctional walls: $(2.49 \pm 0.41$ for nondysfunctional vs., $1.55 \pm 0.63$ in dysfunctional areas, $\mathrm{p}<0.0001$ ). However, There was no significant difference in the mean TDI at rest for viable and non-viable walls as detected by dobutamine stress echocardiography $(5.83 \pm 0.73$ in viable vs. $5.85 \pm 0.76$ in nonviable walls, with $\mathrm{p}=0.9$ non-significant). But the increase in TDI velocity with peak dobutamine was significantly more in viable $(1.97 \pm 0.44)$ vs. non-viable $(1.14 \pm 0.54)$ walls, with $\mathrm{p}<0.0001$, indicating that these regions were hibernating.

\section{The same findings were found in several studies}

Bountioukos $\mathrm{M}$ et al., ${ }^{47}$ in a study on 93 pt., Pulsed-wave TDI at rest demonstrated that dysfunctional regions had lower systolic velocities compared with non-dysfunctional regions $(6.2 \pm 1.9 \mathrm{~cm} / \mathrm{sec}$ vs. $7.1 \pm 1.8 \mathrm{~cm} / \mathrm{sec}, \mathrm{P}<0.001)$. There was no difference in systolic 
velocity at rest between viable and nonviable regions $(6.3 \pm 1.9 \mathrm{~cm} /$ sec vs. $6.3 \pm 1.9 \mathrm{~cm} / \mathrm{sec}$, respectively, $\mathrm{P}<0.93)$. However, during lowdose dobutamine infusion, systolic velocity was significantly higher in viable regions $(8.5 \pm 2.7 \mathrm{~cm} / \mathrm{sec}$ vs $7.8 \pm 2.4 \mathrm{~cm} / \mathrm{sec}$, respectively, $\mathrm{P}<0.002) .{ }^{47}$ In another study by Bountioukos $\mathrm{M}$ et al. ${ }^{47}$ on $70 \mathrm{pt}$ with ICM,. Myocardial systolic velocity of normal or mildly hypokinetic regions (non-dysfunctional regions) was $6.8 \pm 2.0 \mathrm{~cm} / \mathrm{sec}$ at rest and $9.2 \pm(3.3) \mathrm{cm} / \mathrm{sec}$ during low dose dobutamine challenge, while $\Delta \mathrm{Vs}$ was $2.4 \pm 2.8 \mathrm{~cm} / \mathrm{sec}$. Dysfunctional regions had significantly lower velocities: $6.2 \pm 1.6 \mathrm{~cm} / \mathrm{sec}$ at rest $(\mathrm{p}<0.001)$ and $7.6 \pm 2.0 \mathrm{~cm} / \mathrm{sec}$ at low dose dobutamine $(\mathrm{p}<0.001)$, with $\Delta \mathrm{Vs}$ reaching $1.4 \pm 1.5 \mathrm{~cm} / \mathrm{sec}$ ( $\mathrm{p}<0.001)$. In dysfunctional regions with contractile reserve during low dose dobutamine challenge, maximum Vs was $6.1 \pm 1.6 \mathrm{~cm} / \mathrm{sec}$ at rest and $8.0 \pm 2.2 \mathrm{~cm} / \mathrm{sec}$ during low dose dobutamine, and $\Delta V \mathrm{Vs}$ was $1.9 \pm 2.1 \mathrm{~cm} / \mathrm{sec}$. In dysfunctional regions without contractile reserve, Vs was $6.2 \pm 2.0 \mathrm{~cm} / \mathrm{sec}$ at rest $(\mathrm{p}=0.41)$ and $7.3 \pm 2.2 \mathrm{~cm} / \mathrm{sec}$ during low dose dobutamine $(\mathrm{p}=0.04)$, and $\Delta$ Vs was $1.1 \pm 2.0 \mathrm{~cm} / \mathrm{sec}(\mathrm{p}=$ $0.008) .^{30}$ These results were also found in other studies..$^{32,37,38,48}$

\section{Cutoff value for viability}

In the present study, a cut off value of $\geq 1 \mathrm{~cm} / \mathrm{sec}$ in systolic ejection velocity (Vs) at low dose dobutamine infusion was used to define viability of dysfunctional regions. This value was calculated as the best significant value using ROC method. This resulted in 115 $(79.3 \%)$ walls to be diagnosed as viable by this method, while 30 walls were nonviable $(20.7 \%)$

This cutoff value was used by Bountioukos $\mathrm{M}$ et al. ${ }^{38}$, and resulted in the detection of $69.1 \%$ of all dysfunctional regions as viable. (60\% and $73 \%$ of Q wave and nonQ wave dysfunctional regions respectively) ${ }^{38}$. Altinmakas $\mathrm{S}$ et al. ${ }^{49}$ also used similar procedure and assuming 35\% as a cut-off for viability the increase in Systolic velocities by DSE yielded an $89 \%$ sensitivity and $86 \%$ specificity for predicting post-revascularization functional recovery. ${ }^{49}$

Aggeli C et al., ${ }^{41}$ Using ROC curves, the optimal cut-off value for viability assessment was an increase of $0.5 \mathrm{~cm} / \mathrm{sec}$ in ejection velocity (S wave) during LDDSE ( $80 \%$ sensitivity and $88 \%$ specificity, area under the curve 0.80$)^{41}$

Rambaldi R et al., ${ }^{32}$ used an improvement of velocity of $1 \pm 0.5 \mathrm{~cm} /$ $\mathrm{sec}$ as an index of viability. Pulsed-wave Doppler tissue sampling has a sensitivity of $87 \%$, and a specificity of $52 \%$ for the prediction of viable myocardium. This increase exhibited an incremental value to DSE for the diagnosis of myocardial viability. The sensitivity of DSE and pulsed-wave Doppler tissue sampling was $75 / 87 \%$, respectively $(\mathrm{P}<0.05)$, and specificity was $51 / 52 \%$, respectively $(\mathrm{P}=\mathrm{ns}) .^{32}$

In the present study, we did not concentrate on the diastolic indices that can be detected by TDI. This is partially because such indices were not consistently shown to be correlated with viability post-revascularization. Rambaldi $\mathrm{R}$ et al., ${ }^{32}$ found that E/A ratio changes from rest to low dose failed to predict myocardial viability. ${ }^{32}$ Similarly, Bountiuokos $\mathrm{M}$ et al., ${ }^{30}$ found no difference between viable and non viable regions with respect to late diastolic velocities at rest and low dose dobutamine. In addition, early diastolic velocities were more age-dependent than systolic velocities. ${ }^{30}$

The main striking finding of the present study is the improvement of 74 walls of 80 combined TDI \& DSE positive walls, making a sensitivity of $90.2 \%$, similarly 24 of 27 concordant TDI \& DSE negative walls did not improve, making a specificity of $92.3 \%$. This finding may explained by the amplification effect of basal assessment by pulsed-wave Doppler tissue sampling, which is able to detect even small amounts of viable myocardium disseminated between the base and apex. These findings are consistent with higher myocardial integrity required for an inotropic response than for metabolic uptake. $^{50}$

Pulsed wave TDI is a feasible and relatively inexpensive technique that can increase the sensitivity of the dobutamine stress test. It appeared to be minimally influenced by loading changes, when compared to standard echo images in case of doubtful viability assessment, due to suboptimal thickness/texture detection.

\section{Conclusion}

In many of patients with $\mathrm{CAD}$, the extent of remaining viable tissue is of clinical and prognostic significance. It can help to decide between revascularization and cardiac transplantation. Many subjects with heart failure and underlying coronary artery disease have an important amount of viable but dysfunctional myocardium, where myocardium keeps the ability to contract if perfusion improves. The dysfunctional viable myocardium has unique characteristics which form the basis for the different imaging modalities that are currently available for the assessment of myocardial viability. Recent studies showed that the presence of viable myocardium was associated with a greater likelihood of survival in patients with CAD and LV dysfunction, but the assessment of myocardial viability did not identify patients with survival benefit from $\mathrm{CABG}$, as compared with medical therapy alone. Dobutamine stress echocardiography is the most frequently used agent in this setting to assess jeopardized myocardium for viability. Viability is shown by noting improved contraction of a dysfunctional LV wall segment with low-dose dobutamine infusion, which provides adrenergic stimulation. However, to overcome the subjective visual evaluation of regional wall motion, new technologies have been modified; one of these is to assess mitral annular velocity using tissue Doppler imaging. The current study confirmed the importance of using TDI in different mitral annular sites, as an objective tool in detecting myocardial viability with DSE. Pulsed wave TDI is a feasible and relatively inexpensive technique that can increase the sensitivity of the dobutamine stress test. It appeared to be minimally influenced by loading changes, when compared to standard echo images in case of doubtful viability assessment, due to suboptimal thickness/texture detection.

\section{Acknowledgments}

None.

\section{Conflicts of interest}

Author declares there are no conflicts of interest.

\section{Funding}

None.

\section{References}

1. Rosamond W, Flegal K, Furie K, et al. Heart disease and stroke statistics-2008 update: a report from the American Heart Association Statistics Committee and Stroke Statistics Subcommittee. Circulation. 2008;117(4):e25-e146.

2. Bonow R, Dilsizian V, Cuocolo A, et al. Identification of viable myocardium in patients with chronic coronary artery disease and left ventricular dysfunction. Comparison of thallium scintigraphy with reinjection and PET imaging with 18F- fluorodeoxyglucose. Circulation. 1991;83(1):26-37.

3. Diamond GA, Forrester JS, DeLuz PL, et al. Post-extrasystolic potentiation of ischemic myocardium by atrial stimulation. Am Heart J . 1978;95(2):204-209. 
4. Rahimtoola SH. A perspective of the three multicentre randomized clinical trials of coronary bypass surgery for chronic stable angina. Circulation. 1985;72(6 Pt 2):V123 -V135.

5. Braunwald E, Rutherford JD. Reversible ischemic left ventricular dysfunction: evidence for "hibernating myocardium". J Am Coll Cardiol. 1986;8(6):1467-1470.

6. Redwood S, Ferrari R, Marber MS. Myocardial hibernation and stunning: from physiological principles to clinical practice. Heart 1998;80(3):218-222.

7. Page B, Young R, Iyer V, et al. Persistent regional down regulation in mitochondrial enzymes and upregulation of stress proteins in swine with chronic hibernating myocardium. Circ Res. 2008;102(1):103-112.

8. Depre C, Vatner S. Cardio protection in stunned and hibernating myocardium. Heart Fail Rev. 2007;12(3-4):307-317.

9. Depre C, Kim S, John AS, et al. Program of cell survival underlying human and experimental hibernating myocardium. Circ Res 2004;95(4):433-440.

10. Slezak J, Tribulova N, Okruhlicova L, et al. Hibernating myocardium: pathophysiology, diagnosis, and treatment. Can J Physiol Pharmacol. 2009;87(4):252-265.

11. Ross J. Myocardial perfusion-contraction matching. Implications for coronary heart disease and hibernation. Circulation. 1991;83(3):1076-1083.

12. Kelly RF, Sluiter W, McFalls EO. Hibernating myocardium: is the program to survive a pathway to failure? Circ Res. 2008;102:3-5.

13. Schinkel AF, Valkema R, Geleijnse ML, et al. Single-photon emission computed tomography for assessment of myocardial viability. Euro Intervention. 2010;6 Suppl G:G115-G122.

14. Caner B, Beller GA. Are Technetium-99m-Labeled Myocardial Perfusion Agents Adequate for Detection of Myocardial Viability? Clin Cardiol. 1998;21:235-242.

15. Tajouri TH, Chareonthaitawee P. Myocardial viability imaging and revascularization in chronic ischemic left ventricular systolic dysfunction. Expert Rev Cardiovasc Ther. 2010;8(1):55-63.

16. Schinkel AF, Bax JJ, Poldermans D. Clinical assessment of myocardial hibernation. Heart.2005;91(1):111-117.

17. Schinkel AF, Bax JJ, Poldermans D, et al. Hibernating Myocardium: Diagnosis and Patient Outcomes. Curr Probl Cardiol. 2007; 32(7):375-410.

18. Bax JJ, Poldermans D, Elhendy A, et al. Sensitivity, specificity and predictive accuracies of various non-invasive techniques for detecting hibernating myocardium. Curr Probl Cardiol. 2001;26(2):147-186.

19. Allman KC, Shaw LJ, Hachamovitch R, et al. Myocardial Viability Testing and Impact of Revascularization on Prognosis in Patients With Coronary Artery Disease and Left Ventricular Dysfunction: A MetaAnalysis. J Am Coll Cardiol. 2002;39(7):1151-1158.

20. Ong AT, Serruys PW. Complete revascularization: coronary artery bypasses graft surgery versus percutaneous coronary intervention. Circulation. 2006;114(3):249-255.

21. Camici PG, Prasad SK, Rimoldi OE. Stunning, Hibernation, and Assessment of Myocardial Viability. Circulation. 2008;117(1):103-114.

22. Bonow RO, Maurer G, Lee KL, et al. (for the STICH Trial Investigators). Myocardial Viability and Survival in ischemic Left Ventricular Dysfunction. N Engl J Med. 2011;364(17):1617-1625.

23. Gerber BL, Rousseau MF, Ahn SA, et al. Prognostic value of myocardial viability by delayed-enhanced magnetic resonance in patients with coronary artery disease and low ejection fraction: impact of revascularization therapy. J Am Coll Cardiol. 2012;59(9):825-835.
24. Piérard L, Picano E. Myocardial Viability. IN: Eugenio Picano (Ed). Stress Echocardiography. Springer Verlag Berlin Heidelberg. 2009;273-294.

25. Einstein AJ, Moser KW, Thompson RC, et al. Radiation Dose to patients from Cardiac Diagnostic Imaging. Circulation. 2007; 116(11):1290-1305.

26. Perrone-Filardi P, Pace L, Prastaro $M$, et al. Dobutamine echocardiography predicts improvement of hypoperfused dysfunctional myocardium after revascularization in patients with coronary artery disease. Circulation. 1995;91(10):2556-2565.

27. Haque T, Furukawa T, Takahashi M, et al. Identification of hibernating myocardium by dobutamine stress echocardiography: comparison with thallium-201 reinjection imaging. Am Heart J. 1995;130(3 Pt 1):553-563.

28. Hanekom L, Jenkins C Jeffries L, Case C, et al. Incremental Value of Strain Rate Analysis as an Adjunct to Wall-Motion Scoring for Assessment of Myocardial Viability by Dobutamine Echocardiography A Follow-Up Study After Revascularization. Circulation . 2005;112(25):3892-3900.

29. Hoffmann R, Altiok E, Nowak B, et al. Strain rate measurement by Doppler echocardiography allows improved assessment of myocardial viability in patients with depressed left ventricular function. $J$ Am Coll Cardiol. 2002;39(3):443-449.

30. Bountioukos M, Schinkel AF, Bax JJ, et al. Pulsed-wave tissue Doppler quantification of systolic and diastolic function of viable and nonviable myocardium in patients with ischemic cardiomyopathy. Am Heart $J$. 2004;148(6):1079-1084.

31. Yang HS, Kang SJ, Song JK, et al. Diagnosis of viable myocardium using velocity data of Doppler myocardial imaging: comparison with positron emission tomography. J Am Soc Echocardiogr. 2004;17(9):933-940.

32. Rambaldi R, Poldermans D, Bax JJ, et al. Doppler tissue velocity sampling improves diagnostic accuracy during dobutamine stress echocardiography for the assessment of viable myocardium in patients with severe left ventricular dysfunction. Eur Heart J. 2000; 21(13):1091-1098

33. Greenberg N, Firstenberg MS, Castro PL, et al. Doppler-derived myocardial systolic strain rate is a strong index of left ventricular contractility. Circulation. 2002;105(1):99-105.

34. Lang RM, Bierig M, Devereux RB, et al. Recommendations for chamber quantification: a report from the American Society of Echocardiography's Guidelines and Standards Committee and the Chamber Quantification Writing Group, developed in conjunction with the European Association of Echocardiography, a branch of the European Society of Cardiology. $J$ Am Soc Echocardiogr. 2005; 18(12):1440-1463.

35. Bax JJ, Poldermans D, Schinkel AF, et al. Perfusion and contractile reserve in chronic dysfunctional myocardium: relation to functional outcome after surgical revascularization. Circulation. 2002;106(12 suppl I):I14-I18.

36. Carluccio E, Biagioli P Alunni G, Murrone A, et al. Effect of revascularizing viable myocardium on left ventricular diastolic function in patients with ischaemic cardiomyopathy. Eur Heart J. 2009;30(12):1501-1509.

37. Matsuoka M, Oki T, Mishiro Y, et al. Early systolic mitral annular motion velocities responses to dobutamine infusion predict myocardial viability in patients with previous myocardial infarction. Am Heart $J$. 2002;143(3):552-558.

38. Bountioukos M, Schinkel A, Bax JJ, et al. Quantification of regional left ventricular function in $\mathrm{Q}$ wave and non-Q wave dysfunctional regions by tissue Doppler imaging in patients with ischaemic cardiomyopathy. Heart. 2003;89(11):1322-1326. 
39. Carluccio E, Biagioli P, Alunni G, et al. Patients With Hibernating Myocardium Show Altered Left Ventricular Volumes and Shape, Which Revert After revascularization. Evidence That Dyssynergy Might Directly Induce Cardiac Remodeling. J Am Coll Cardiol . 2006;47(5):969-977.

40. La Canna G, Rahimtoola SH, Visioli O, et al. Sensitivity, specificity, and predictive accuracies of non-invasive tests, singly and in combination, for diagnosis of hibernating myocardium. Eur Heart $J$. 2000;21(16):1358-1367

41. Aggeli C, Giannopoulos G, Roussakis G, et al. Pre-Ejection TissueDoppler Velocity Changes During Low Dose Dobutamine Stress Predict Segmental Myocardial Viability. Hellenic J Cardiol. 2007;48(1):23-29.

42. Tani T, Teragaki M, Watanabe H, et al. Prediction of Functional Recovery in Patients With Myocardial Infarction After Revascularization Comparison of Low-Dose Dobutamine Stress Echocardiography With Fluorine-18 Fluorodeoxyglucose Positron Emission Tomography. Jpn Circ J. 2001;65(3):177-181.

43. Karabinos I, Papadopoulos A, Karvouni E, et al. Reliability and Safety of Dobutamine Stress Echocardiography for Detection of Myocardial Ischemia-Viability: Experience From 802 Consecutive Studies. Hellenic J Cardiol. 2004;45:71-83.

44. Ausma J, Schaart G, Thone F, et al. Chronic ischemic viable myocardium in man: aspects of dedifferentiation. Cardiovasc Pathol. 1995;4(1):29-37.
45. Vanoverschelde JL, Melin JA, Depré C. Time-course of functional recovery of hibernating myocardium after coronary revascularization. Circulation. 1994;90(pt 2):1-378.

46. Shivalkar B, Maes A, Borgers M, et al. Only hibernating myocardium invariably shows early recovery after coronary revascularization. Circulation. 1996;94(3):308-315.

47. Bountioukos M, Schinkel AF, Bax JJ, et al. Pulsed-wave tissue Doppler quantification of systolic and diastolic function of viable and nonviable myocardium in patients with ischemic cardiomyopathy. Am Heart $J$. 2004;148(6):1079-1084.

48. Gorcsan J $3^{\text {rd }}$, Deswal A, Mankad S, et al. Quantification of the myocardial response to low-dose dobutamine using tissue Doppler echocardiographic measures of velocity and velocity gradient. $\mathrm{Am} \mathrm{J}$ Cardiol. 1998;81(5):615-623.

49. Altinmakas S, Dagdeviren B, Uyan C, et al. Prediction of viability by pulsed-wave Doppler tissue sampling of asynergic myocardium during low-dose dobutamine challenge. Int J Cardiol. 2000;74(2-3):107-113.

50. Shabana A, El-Menyar A. Myocardial viability: what we knew and what is new. Cardiol Res Pract. 2012;2012:607486. 